\title{
El autoconcepto físico de jóvenes futbolistas de alto rendimiento
}

\section{High-performance football young player's physical self-concept}

\section{O autoconceito físico de jovens jogadores de futebol de alto rendimento}

\author{
Bingen Aróstegi, Alfredo Goñi, Asier Zubillaga y Guillermo Infante
}

Universidad del País Vasco, UPVIEHU

\begin{abstract}
Resumen: Este estudio tiene por objeto precisar diferencias en el autoconcepto físico entre futbolistas jóvenes, noveles y veteranos, de alto rendimiento y estudiantes de su misma edad. Participan en el estudio 216 personas (114 futbolistas de alto rendimiento y 112 estudiantes que no tienen la condición de ser futbolistas de élite) de entre 14 y 23 ańos; los futbolistas, se dividen, a su vez, en 59 noveles (de 14 a 17 ańos) y 55 veteranos (de 18 a 23 años). Para la medición del autoconcepto se utilizó el Cuestionario de Autoconcepto Físico (CAF), de Goñi, Ruiz de Azúa y Rodríguez (2006). Los resultados ponen de manifiesto que el grupo de futbolistas de élite obtiene puntuaciones más altas, estadísticamente significativas, que el grupo de no-futbolistas en las autopercepciones de habilidad, condición, atractivo y fuerza así como en el autoconcepto físico general pero no en el autoconcepto general; no se verifican, sin embargo, diferencias entre futbolistas de élite noveles y veteranos.
\end{abstract}

Palabras clave: Autoconcepto físico, futbol, jugadores de alto rendimiento. Abstract:: This study is intended to clarify differences in physical self-concept among high-performance football young players and students of the same age. Participating in the study 216 people (114 players, high-performance 112 students who have no status as elite players,) between 14 and 23 years; the players are divided, in turn, 59 junior and 55 senior, corresponding to the 14 age group to 17 and 18 to 23 years. For the measurement of self-concept questionnaire was used physical self (CAF), Goñi, Ruiz de Azua and Rodriguez (2006), composed of the following scales: physical ability, physical condition, physical attractiveness, physical strength and general physical self-concept as general self-concept. The results show that the group of elite players gets higher scores, statistically significant, than the group of non-elite players in five of the six dimensions of physical selfconcept: skill, fitness, attractiveness, strength and self general physical selfconcept but not in general, however, there are not differences between elite novel players and elite veteran players.

Keywords: Physical self-concept, soccer, high-performance players.

Resumo: Este estudo tem o objetivo de marcar as diferenças no autoconceito físico entre jovens jogadores, novatos e veteranos, de alto rendimento e estudantes de uma mesma idade. Participaram nesse estudo 216 pessoas (114 jogadores de futebol de alto rendimento e 112 estudantes que no eram jogadores de futebol de elite) entre 14 e 3 anos de idade; os jogadores de futebol se dividiam em 59 novatos ( 14 a 17 anos) e 55 veteranos (18 a 23 anos). Para medir o autoconceito foi utilizado o Questionário de Autoconceito Físico (CAF), Gońi, Ruiz de Azua y Rodriguez (2006). Os resultados manifestam que o grupo de jogadores de futebol de elite obtém pontuaçóes mais altas estatisticamente significativas que o grupo de não jogadores de futebol nas autopercepçôes de habilidade, condição, atrativo e força, assim como no autoconceito físico geral, mas no autoconceito geral náo foram encontradas diferenças entre jogadores de futebol de elite, novatos e veteranos.

Palavras Chave: autoconceito físico, futebol, jogadores de alto rendimento.

\section{Introducción}

El autoconcepto es un rasgo de gran importancia psicológica dadas las vinculaciones que mantiene con numerosas variables psicosociales y también por mostrarse modificable mediante una adecuada intervención (Madariaga y Goñi, 2009). El autoconcepto físico es uno de los principales dominios del autoconcepto, entendido desde una perspectiva jerárquica y multidimensional (Shavelson, Hubner y Stanton, 1976) siendo el modelo tetradimensional del autoconcepto físico (Fox y Corbin, 1989; Goñi, Ruiz de Azúa y Rodríguez, 2006), que incluye las dimensiones de habilidad, condición, atractivo y fuerza, el más ampliamente aceptado en nuestros días.

Correspondencia: Guillermo Infante Borinaga. Universidad del País Vasco. Escuela Universitaria de Magisterio de Vitoria-Gasteiz. Juan Ibañez de Santo Domingo, 1 C.P. 01006, Vitoria- Gasteiz.

guillermo.infante@ehu.es

Nota: Esta investigación se ha realizado dentro del proyecto EDU200910102 (subprograma EDUC), subvencionado por el MICINN.
El autoconcepto físico guarda relación con una amplia nómina de variables psicosociales como, por ejemplo, los hábitos de conducta alimentaria (Goñi y Rodríguez, 2004), la actividad física tanto en la juventud (Candel, Olmedilla y Blas, 2008; Crocker, Sabiston, Kowalski, McDonough y Kowalski, 2006; Welk y Eklund, 2005) como en la edad adulta (Anderson, Murphy, Murtagh y Nevill, 2006; Elavski, 2010; Esnaola, 2009; Infante, Goñi, y Villarroel, 2011) o el bienestar/malestar psicológico (Rodríguez, 2009). Asimismo se relaciona directamente con variables biológicas como la tensión arterial (Urdampilleta, González, Infante y Goñi, 2011).

En todo caso, y hasta el presente, la abundante información disponible (Goñi, 2009) sobre diferencias en el autoconcepto físico se ha obtenido mediante estudios con participantes de la población general lo que significa que apenas se sabe nada sobre el autoconcepto físico de personas con deficiencias físicas o con altas capacidades físicas, como pueden ser los de- 
portistas de élite. La excepción la constituyen sendos estudios recientes que han utilizado el Cuestionario de Autoconcepto Físico (CAF) de Goñi et al. (2006). En uno de ellos, se comprueba $n$ que los fisioculturistas ofrecen índices estadísticamente más altos que el colectivo de usuarios de sala de musculación en todas las escalas, lo que avalaría la tesis de que a mayor intensidad de práctica deportiva corresponde un mejor autoconcepto físico incluso dentro del colectivo de personas que desarrollan altos niveles de actividad física (GonzálezMartí, 2012). Sin embargo, otro estudio (Axpe, 2012) pone de manifiesto que el autoconcepto físico de los estudiantes de una Facultad de Ciencias de la Actividad Física es inferior al del alumnado de otras titulaciones del mismo campus, lo que induce a una conclusión inversa a la anterior.

En definitiva, se sabe que, dentro de la población general, la práctica físico-deportiva guarda directa relación con el autoconcepto físico, con independencia del deporte que se realice pero asociada a su frecuencia (recomendable la frecuencia alta). En cambio, hay datos poco consistentes y escasos respecto a la relación entre el autoconcepto y la mayor o menor intensidad de la actividad física de deportistas de élite.

El objetivo de este trabajo es doble: a. identificar diferencias en el autoconcepto físico entre jóvenes futbolistas de elite y estudiantes no pertenecientes a un grupo de elite; b. precisar posibles diferencias dentro del colectivo de futbolistas de élite. Las expectativas de resultados son que: (1) el autoconcepto físico de los futbolistas de élite sea superior al de jóvenes que no son de élite; (2) el autoconcepto físico de los jóvenes futbolistas de élite más consolidados (veteranos) sea superior al de los más noveles.

\section{Método}

\section{Participantes}

Participan en esta investigación 226 sujetos con edades comprendidas entre los 14 y los 23 ańos y pertenecientes a dos grupos diferenciados (tabla 1).

Tabla 1. Número y edad de los participantes en la investigación.

\begin{tabular}{ccccc}
\hline \multirow{3}{*}{ Colegio } & Grupo & N & M & DT \\
\hline \multirow{4}{*}{ Rendimiento } & Veteranos & 28 & 20.82 & 1.68 \\
& Novel & 84 & 15.02 & 1.12 \\
& Veterano & 59 & 19.14 & 1.51 \\
& Novel & 55 & 15.05 & 1.46 \\
& Colegio & 112 & 16.47 & 2.83 \\
& Rendimiento & 114 & 17.17 & 2.42 \\
\hline & Total muestra & $\mathbf{2 2 6}$ & & \\
\hline
\end{tabular}

Por un lado, 114 sujetos pertenecen a la estructura deportiva de un club de fútbol de alto rendimiento, en sus diferentes categorías de cadete B (nacidos en 1997), cadete A (nacidos en 1996), juvenil B (nacidos en 1995 y 1994), juvenil A (nacidos en 1995, 1994 y 1993), Baskonia (nacidos en 1990, 1991 y 1992) y Bilbao Athletic (nacidos entre 1989 y 1993). Conforman estos futbolistas de alto rendimiento dos grupos: noveles $\mathrm{y}$ veteranos. Se considera futbolistas noveles a los deportistas pertenecientes a los equipos cadete $B$, cadete $A$ y juvenil $B$, es decir los más jóvenes $(\mathrm{N}=55)$; e integran el grupo de futbolistas veteranos los jugadores de mayor edad dentro de grupo de rendimiento y pertenecientes al equipo juvenil A, al Baskonia $\mathrm{y}$ al Bilbao Athletic $(\mathrm{N}=59)$.

Por otro lado, 112 alumnos son escolares del colegio El Salvador Maristas de Bilbao divididos por nivel escolar ESO nivel 3 (nacidos en 1996), ESO nivel 4 (nacidos en 1995), bachiller 1 (nacidos en 1994) bachiller 2 (nacidos en 1993 y 1992) y alumnos pertenecientes al segundo nivel de la Escuela de Magisterio de la UPV-EHU de Vitoria-Gasteiz (nacidos entre 1989 y 1993). Ninguno de estos escolares es futbolista de alto rendimiento.

\section{Variable e instrumentos de medida}

La variable teórica de esta investigación es el autoconcepto físico, que se mide mediante el Cuestionario de Autoconcepto Físico (CAF), el cual consta de las siguientes escalas:

A. Habilidad física ( $H$ ). Percepción de las cualidades ("soy bueno, tengo cualidades") y habilidades ("me veo hábil, me veo desenvuelto") para la práctica de actividad física y deportiva, capacidad de aprender deportes; seguridad personal y predisposición ante los deportes.

B. Condición física. (C). Normalmente la condición física se suele expresar por el nivel de forma física que demostremos. La definición de forma física es la siguiente, "nivel de energía y vitalidad que permite a las personas llevar a cabo las tareas diarias habituales, disfrutar del tiempo de ocio activo y afrontar las emergencias imprevistas sin fatiga excesiva, a la vez que ayuda a evitar las enfermedades derivadas de la falta de actividad física y a desarrollar al máximo la capacidad intelectual". A la hora de realizar determinadas actividades físicas nuestro rendimiento será equivalente al grado de forma física que haya adquirido a través de la práctica. Esto nos permitirá evaluarnos acerca de nuestra resistencia a la hora de aguantar un esfuerzo, ya sea continuo o inmediato.

C. Atractivo físico (A). Viene determinada por la imagen corporal que poseamos se puede diferenciar una interior y una exterior. En la medición de esta variable nos vamos a centrar en nuestra imagen exterior de cara a los demás y a los dictados que marcan la sociedad en 
la que vivimos. La seguridad y confianza que nos va a aportar dicha información será importante en vistas a la elaboración del autoconcepto. De otra parte, la información que nos puede proporcionar la imagen interna nos puede ayudar en la visión que tengamos de nuestra competencia motriz, pero esta queda fuera de nuestro estudio.

D. Fuerza (F). Verse y/o sentirse fuerte, con capacidad para levantar peso, con seguridad ante ejercicios que exigen fuerza y predisposición a realizar dichos ejercicios.

E. Autoconcepto físico general ( $A F G$ ). Opinión y sensaciones positivas (felicidad, satisfacción, orgullo y confianza) en lo físico. Se han utilizado ítems específicos para la medición, dejando de lado las variables anteriormente mencionadas.

F. Autoconcepto general $(A G)$. Grado de satisfacción con uno mismo y con la vida en general. Un autoconcepto positivo constituye un factor fundamental para la consecución de una personalidad integrada, así como para la adquisición y el mantenimiento de patrones conductuales ajustados y saludables.

\section{Procedimiento}

Tras requerir las autorizaciones oportunas de la dirección del club de alto rendimiento y de la dirección y de las familias de los alumnos menores de edad del centro en el que se pasaron las encuestas, los cuestionarios han sido pasados siguiendo el mismo protocolo. En el caso del club de rendimiento se ha utilizado el primer contenido de entrenamiento en cada grupo de individuos, y el caso de los alumnos en la sala de estudio, cada individuo en un pupitre, dándoles un tiempo estimado de veinte minutos para poder realizarlo. Los alumnos del colegio han realizado los cuestionarios en las horas de Educación física.

Todos los cuestionarios han sido leídos en voz alta por el investigador antes de que contestaran a las preguntas. Todos los grupos lo han rellenado por separado y todos los cuestionarios fueron respondidos de forma voluntaria. Previamente se instruyó a los sujetos para que respondieran con la mayor sinceridad posible, garantizándoles en todo momento la confidencialidad de los datos que se obtuvieran.

\section{Análisis de los datos}

Se trata de un estudio de carácter correlacional entre variables en el que se precisan establecer contraste de puntuaciones de tendencia central ( $\mathrm{t}$ de Student). Para el análisis de datos se ha utilizado el programa SPSS en su versión 17.0. En todos los casos el nivel de significación fue establecido en el nivel $\mathrm{p}<0.05$.

\section{Resultados}

En primer lugar, en orden a identificar diferencias en autoconcepto entre los jóvenes futbolistas de élite y escolares que no son futbolistas de élite, se lleva a cabo un contraste de medias, cuyos resultados se presentan en la tabla 2 .

Tabla 2. Diferencias en las respuestas al CAF entre futbolistas de élite y jóvenes de su edad.

\begin{tabular}{cccccc}
\hline CAF & Grupo & M & DT & t & Sig. \\
\hline \multirow{2}{*}{ Habilidad } & Futbolistas & 20.09 & 1.8 & 2.97 & .003 \\
& Colegio & 19.20 & 1.98 & & \\
Condición & Futbolistas & 19.66 & 2.07 & 3.40 & .001 \\
& Colegio & 18.42 & 2.57 & & \\
Atractivo & Futbolistas & 23.76 & 2.56 & 4.30 & .001 \\
& Colegio & 21.37 & 4.05 & & \\
Fuerza & Futbolistas & 21.62 & 3.41 & 3.40 & .001 \\
& Colegio & 19.58 & 4.16 & & \\
A.F.G. & Futbolistas & 20.77 & 1.62 & 2.41 & .017 \\
& Colegio & 19.76 & 2.47 & & \\
A.G. & Futbolistas & 20.97 & 1.93 & & .220 \\
& Colegio & 21.15 & 2.21 & & \\
\hline
\end{tabular}

Se observan diferencias estadísticamente significativas entre el grupo de futbolistas y el grupo de colegio siendo más altas las puntuaciones de los futbolistas en cinco de las seis dimen- siones del CAF: habilidad, condición, atractivo, fuerza y autoconcepto físico general. Estos datos refrendan plenamente la primera expectativa de resultados (mejor autoconcepto físi- 
co de los futbolistas de élite) verificada en las cinco escalas del CAF que miden autoconcepto físico; no se comprueban, en cambio, diferencias en el autoconcepto general, lo cual, como se comentará en la discusión de los resultados, corrobora las presunciones teóricas del modelo jerárquico y multidimensional del autoconcepto.

En la tabla 3 se ofrecen los datos correspondientes a las respuestas de futbolistas noveles y veteranos al CAF.

Tabla 3. Diferencias en las respuestas al CAF entre futbolistas de élite noveles y veteranos.

\begin{tabular}{cccccc}
\hline CAF & Grupo & $\mathbf{M}$ & $\mathrm{DT}$ & $\mathbf{t}$ & Sig. \\
\hline \multirow{2}{*}{ Habilidad } & Veteranos & 27.12 & 2.58 & -0.213 & .832 \\
& Noveles & 27.00 & 2.20 & & \\
Condición & Veteranos & 26.19 & 3.10 & -0.157 & .876 \\
& Noveles & 26.07 & 3.28 & & \\
\multirow{2}{*}{ Atractivo } & Veteranos & 26.48 & 3.28 & -0.193 & .848 \\
& Noveles & 26.33 & 3.41 & & \\
Fuerza & Veteranos & 24.26 & 4.05 & -0.604 & .547 \\
& Noveles & 23.70 & 3.69 & & \\
A.F.G. & Veteranos & 28.00 & 2.21 & -0.538 & .592 \\
& Noveles & 27.70 & 2.61 & & \\
A.G. & Veteranos & 26.77 & 2.55 & 1.445 & .152 \\
& Noveles & 27.59 & 2.15 & & \\
\hline
\end{tabular}

Los datos de la tabla 3 indican que no existen diferencias estadísticamente significativas entre futbolistas noveles y veteranos de alto rendimiento.

\section{Discusión}

En relación con el primer objetivo de este estudio se han comprobado diferencias estadísticamente significativas entre el grupo de rendimiento y el grupo de colegio siendo mayores las distintas medidas de autoconcepto físico del grupo de rendimiento. Se han obtenido valores significativamente más altos en cinco de los seis índices de autopercepción física del CAF: habilidad, condición física, atractivo, fuerza y autoconcepto físico general a favor del grupo de rendimiento mientras que no se observan diferencias significativas en cuanto al autoconcepto general.

Estos resultados son consistentes con el modelo teórico del autoconcepto según el cual el autoconcepto general viene a ser un sumatorio de los autoconceptos en diversos dominios sopesados en función de la importancia conferida a los mismos o por contrabalanceo con el autoconcepto en otros dominios. Es compatible, en consecuencia, un autoconcepto global positivo a pesar de que uno de los dominios del mismo (el físico, por ejemplo, no sea alto) ya que puede estar compensado por el nivel en otros dominios o por la importancia que se otorga a cada uno de ellos.

En todo caso, la aportación más importante deriva de las características de la muestra. Existen muy pocos antecedentes de investigación que comparen segmentos de población privilegiados (superdotación intelectual, física...) con el resto de la población; y, en particular (Kavussanu, White, Jowett, y England, 2011), no existen estudios que comparen el autoconcepto físico de futbolistas de élite con la población general. Esta carencia de datos empíricos es precisamente la que ha alentado a sostener presunciones teóricas contrapuestas respecto a si cabe esperar que los deportistas de élite tengan mejor autoconcepto físico al tener mayores cualidades que la media de la población o que tengan un autoconcepto físico similar ya que su referencia comparativa son los iguales o lo tengan inferior dada su alto nivel de aspiraciones en ese ámbito. Los datos obtenidos en este estudio apuntan en una doble dirección: a. tienen un autoconcepto físico superior al de la población; b. eligen por lo general como término de comparación su propio grupo de referencia.

De todos modos, las conclusiones que puedan extraerse en estos momentos de los datos obtenidos no dejan de tener carácter tentativo ya que el número de los participantes en el estudio es relativamente pequeño y se precisan realizar nuevos estudios que dispongan de un número más amplio de participantes para poder tomar en consideración, con mínimas garantías estadísticas, las diferencias de actividad de los jóvenes no futbolistas-profesionales.

En cuanto al objetivo segundo (precisar diferencias en el autoconcepto entre noveles y veteranos dentro del grupo de 
alto rendimiento) no se han encontrado diferencias estadísticamente significativas entre noveles y veteranos. Los datos de la investigación previa (Goñi, 2009) indican la existencia de una mejora del autoconcepto físico durante estos años a favor de las personas de más edad. Esta pauta no se repite en este estudio lo que pudiera atribuirse al hecho de que el colectivo de los jóvenes futbolistas de alto rendimiento manifiesta un autoconcepto más alto que el de otros jóvenes de su edad y por tanto puede que hayan alcanzado un desarrollo del mismo más prematuro que el de los de su misma edad.

Esta investigación contribuye a consolidar la tesis de que los jóvenes futbolistas de alto rendimiento desarrollan un autoconcepto físico superior al de otros jóvenes de su edad que no pertenecen a su colectivo. Esta constatación avala la interpretación de que no sólo desarrollan un autoconcepto fí- sico alto sino también un autoconcepto físico maduro o sano dado que, por lo que cabe suponer, eligen adecuados términos de comparación y otorgan a lo físico un correcto grado de importancia. Ahora bien, merecería la pena confirmar esta interpretación indagando los mecanismos psicológicos que activan los jóvenes futbolistas en la construcción de su buen autoconcepto físico entre los que figuran las atribuciones, el self-ideal, la importancia conferida a lo físico o la percepción de autoeficacia. El indagar cómo se activan estos mecanismos, con el recurso a metodologías cualitativas, ha de permitir alcanzar una explicación de lo que por el momento puede darse como constatación válida: el mejor autoconcepto físico de los jóvenes futbolistas de élite en comparación con sus compañeros de estudios de su misma edad.

\section{Referencias}

1. Anderson, A. G., Murphy, M. H., Murtagh, E., y Nevill, A. (2006). An 8 -week randomized controlled trial on the effects of brisk walking, and brisk walking with abdominal electrical muscle stimulation on anthropometric, body composition, and self-perception measures in sedentary adult women. Psychology of Sport and Exercise, 7, 437-451.

2. Axpe, I. (2012). Diseño y evaluación de un programa para la mejora del autoconcepto físico. Tesis doctoral. Bilbao: Universidad del País Vasco.

3. Candel, N., Olmedilla, A., y Blas, A. (2008). Relaciones entre la práctica de actividad física y el autoconcepto, la ansiedad y la depresión en chicas adolescentes. Cuadernos de Psicología del Deporte, 8(1), 61-77.

4. Crocker, P. R. E., Sabiston, C. M. Kowalski, K. C., McDonough, M. H., y Kowalski, N. P. (2006). Longitudinal assessment of the relationship between physical self-concept and helath-related behaviour and emotion in adolescenct girls. Journal of Applied of Sport Psychology, 18, 185-200.

5. Esnaola, I. (2009). El autoconcepto físico durante el ciclo vital. Anales de Psicología, 24(1), 1-8.

6. Elavski, S. (2010). Longitudinal examination of the exercise and selfesteem model in middle aged woman. Journal of Sport and Exercise Psychology, 32, 862-880.

7. Fox, K. R., y Corbin, C. B. (1989) The physical Self-Perception Profile: Development and preliminary validation. Journal of Sports and Exercise Psycology, 11, 408-430.

8. González-Martí, I. (2012). Elementos que integran el perfil de la persona afectada por dismorfia muscular (anorexia). Prevalencia del trastorno en fisioculturistas y usuarios de la sala de musculación. Tesis Doctoral. Cuenca: Universidad de Castilla La Mancha.
9. Goñi, A, Ruiz de Azúa, S., y Rodríguez, A. (2006). Cuestionario de Autoconcepto Fisico (CAF). Madrid: EOS.

10. Gonni, A., y Rodríguez, A. (2004). Trastornos de conducta alimentaria, práctica deportiva y autoconcepto físico en adolescentes. Actas Españolas de Psiquiatría, 32(1), 29-36.

11. Gońi, A. (2009). El autoconcepto físico. Madrid: Pirámide.

12. Kavussanu, M., White, S. A., Jowett, S., y England, S. (2011). Elite and no-elite male footballers differ in goal orientation and perceptions of parental climate. International Journal of Sport and Exercise Psychology, 9(3), 284-290.

13. Infante, G., Goñi, A. y Villarroel, J. D. (2011). Actividad física, autoconcepto físico y general a lo largo de la edad adulta. Revista de Psicologia del Deporte, 20(2), 429-444.

14. Madariaga, J. M., y Goñi, A. (2009). El desarrollo psicosocial. Revista de Psicodidáctica, 14(1), 95-118.

15. Rodríguez, A. (2009). Autoconcepto físico y bienestar/malestar físico en la adolescencia. Revista de Psicodidáctica, 14(1), 155-158.

16. Shavelson, R. J., Hubner, J. J., y Stanton, J. C. (1976). Self concept: Validation of construct retations. Review of Educational Research, 46(3), 407-441.

17. Urdampilleta, A., González, J., Infante, G., y Goñi, A. (2011). La tensión arterial sistémica y el autoconcepto físico en la adolescencia. Cuadernos de Psicología del Deporte, 11(2), 25-34.

18. Welk, G. J., y Eklund, B. (2005). Validation of the children and youth physical self-perceptions profile for young children. Psychology of Sport and Exercise, 6, 51-65. 